\title{
Advances in dissolution understanding and their implications for cement hydration
}

\author{
Patrick Juilland ${ }^{\mathrm{a}}{ }^{*}$, Luc Nicoleau ${ }^{\mathrm{b}}$, Rolf S. Arvidson ${ }^{\mathrm{c}}$, Emmanuel Gallucci $^{\mathrm{a}}$ \\ Sika Technology AG, Zürich, Switzerland \\ b Placoplatre, Vaujours, France \\ c University of Bremen, Bremen, Germany
}

Received: 08 December 2017 / Accepted: 28 December 2017 / Published online: 30 December 2017

(C) The Author(s) 2017. This article is published with open access and licensed under a Creative Commons Attribution 4.0 International License.

\begin{abstract}
Recent advances in bridging kinetics and thermodynamics of mineral dissolution have opened new horizons in our understanding of the role of dissolution in cement hydration. Indeed most hydration kinetic regimes of alite can be rationally envisioned from a dissolution perspective. This short note reviews some key findings on dissolution mechanisms and their implication for cementitious systems.
\end{abstract}

Keywords: Carbonation; Slags; CO2; Aggregates; Compacts; Curing

\section{Introduction}

In the past several decades there has been significant progress in our understanding of mineral reactions, particularly in terms of their chemical kinetics and thermodynamics. This understanding has come about because of key advances in instrumental and analytical sciences, coupled with parallel strides in theoretical understanding. These advances yield important potential benefits for applied material science fields as well, particularly those involving dissolution in under-saturated systems.

Until very recently, the dissolution of alite, impure $\mathrm{Ca}_{3} \mathrm{SiO}_{5}$ ( $C_{3} S$ in cement shorthand), the main phase of modern cement, has nearly always been neglected or simplistically accounted for in all models addressing cement hydration kinetics. This problematic treatment has two origins: first, experimental and technical limitations have strongly prevented studying the dissolution step in isolation, independently from the other mechanisms occurring during cement hydration. Second, many models have relied essentially on C-S-H (the main hydrate resulting from the $\mathrm{C}_{3} \mathrm{~S}$ hydration) precipitation to describe the evolution of the degree of hydration (DoH), obviating the need to properly describe dissolution. Since $\mathrm{C}_{3} \mathrm{~S}$ dissolution and the precipitation of $\mathrm{C}-\mathrm{S}-\mathrm{H}$ and Portlandite share the same ions, they are kinetically coupled through their degrees of under- and super-saturation. Elaborating kinetics models that neglect or simplify dissolution is therefore highly tentative, in light of experimental observations showing the relationship hydration / intrinsic $\mathrm{C}_{3} \mathrm{~S}$ reactivity, impacted for example by particle size [1], annealing [2], or other thermal or mechanical treatments $[3,4]$.

Guided by the knowledge gained in mineral dissolution, pioneering works $[2,5]$ have tried to remedy this unsatisfying situation and highlighted the crucial role of dissolution within the different hydration kinetic steps, still the subject of controversial debates within the cement science community. This review summarizes some works in geochemistry and concepts relevant to the dissolution of under-saturated systems, bearing both important commonalities and differences, how this understanding was recently transferred and integrated in the case of alite, the subsequent implications for cement hydration, and the remaining controversial issues. Those recent scientific advances definitively open new horizons, but the journey remains long before implementing an exact dissolution model as a building block of cement hydration kinetics, and the best attempt so far remains unsatisfactory [6]. 


\section{Review of progress in geochemical kinetics relevant to cementitious systems}

\subsection{Early advances: how do crystal defects control mineral dissolution rate?}

Crystal dissolution dynamics in minerals are inexorably bound to the distribution of their defects, particularly screw (and edge) dislocations. Such defects arise during crystal growth (e.g., either during high temperature crystallization from a melt, precipitation from a low temperature solution, by phase inversions or related reactions) or alternatively, via subsequent (plastic) deformation. Dislocations may be categorically characterized by the Burgers vector, $\boldsymbol{b}$, which describes the internal displacement of the lattice by the dislocation itself (relevant review in [7]). A key insight into the relationship between crystal stability and dissolution mechanism of crystals came with Frank's [8] recognition that screw dislocations in minerals of appropriate physical characteristics (sufficient elastic moduli, surface energy, and Burgers vector modulus) would give rise to so-called hollow cores (now readily visible by AFM $[9,10,11]$ ). These are spontaneously developed capillaries surrounding the dislocation axis, reflecting an energetic balance between the excess strain energy contributed by the dislocation itself and the positive surface work expended in expansion of the capillary's interior surface. Frank derived the radius of the hollow core ( $r_{0}$, Frank's radius) as

$$
r_{0}=\frac{\mu}{\gamma} \frac{b^{2}}{8 \pi^{2}}
$$

where $b$ is the modulus of the Burgers vector, $\gamma$ the surface energy, and $\mu$ the shear modulus. Although screw dislocations were a central focus of the landmark crystal growth paper by Burton, Cabrera, and Frank [12], these features also play a key role in etch pit development - and thus crystal dissolution. This role was recognized by Cabrera and Levine [13], who, in deriving solutions for the general equation for the advance of spiral step, showed that beyond a critical under-saturation (see $\Delta G_{\text {crit }}$ below) no stable, steady state spirals could exist. At this boundary, the hollow core of a screw dislocation emerging at the crystal surface would become unstable, enlarging its radius at its outcrop and developing into a macroscopic etch pit. Cabrera and Levine represented this under-saturation limit as a dimensionless ratio of $r_{0}$ with that of the so-called critical nucleus for 2D nucleation: $s_{0}^{*}=r_{0} / \rho_{c}^{*} \cong-\frac{1}{4}$ (where $\rho_{c}$ is the critical radius of a hole, and thus negative). The critical radius is in turn sensitive to free energy:

$$
\rho_{c}=\frac{\gamma \bar{V}}{\Delta \mu_{0}}
$$

Here $\bar{V}$ is the molar volume of the crystal, and $\Delta \mu_{0}$ the chemical potential difference between the crystal and its coexisting solution or vapor. This paper thus established the key relationships between the development of surface etch pits in minerals, hollow core stability, and free energy. Closer to equilibrium or under supersaturated conditions $\left(r_{0} / \rho_{c}>\right.$ 0 ), hollow cores would be theoretically stable. Subsequent work by van der Hoek and coworkers [14] further explored this approach and showed how a phase map of hollow core stability could be defined, permitting broad distinction among potential dissolution mechanisms in minerals as a function of free energy, dislocation parameters, and elastic properties. This work also demonstrated that certain crystals may simply be incapable of sufficient strain energy distribution to support hollow cores; in these materials, etch pits may form, but without the mechanistic relationship to capillary formation.

Those concepts established a basic theoretical framework for the relationship between defect character and etch pit development. Lasaga and Blum [15] showed how this energetic framework, combined with kinetic Monte Carlo simulations to compute dissolution rates, could be applied to actual minerals. Consistent with work cited above, they established the existence of a free energy limit with respect to the bulk crystal,

$$
\Delta G_{\text {crit }}=-\frac{2 \pi^{2} \gamma^{2} \bar{V} \kappa}{\mu b^{2}}
$$

Here $\kappa$ is a parameter describing the contribution of screw, $\kappa=1$, versus edge, $\kappa=1-v$ ( $v$ is Poisson's ratio), to dislocation geometry. These theoretical advances set the stage for a major advance in crystal dissolution theory: the stepwave model of Lasaga and Luttge [16]. This work incorporated the previous relationships, proposing a general dependence of crystal dissolution rate on free energy:

$$
\begin{aligned}
& \qquad r=A\left(1-e^{\Delta G / k T}\right) \tanh \left[\frac{B}{f(\Delta \mathrm{G})}\right] f(\Delta \mathrm{G}), \\
& \text { where } f(\Delta \mathrm{G}) \equiv 1-\frac{1-e^{\Delta G / k T}}{1-e^{\Delta G} \mathrm{crit} / k T}
\end{aligned}
$$

The model provided two critical conceptual advances. First it revealed the true relationship between etch pit nucleation and overall dissolution rate: heretofore, etch pits were regarded as the obvious centers of all dissolution activity. Other areas of the crystal surface such as terraces were thought to be largely passive and dormant. This assumption gives rise to the pejorative distinction between "reactive" versus "non-reactive" surface area. The stepwave model refuted this distinction, showing how chains of steps ("stepwaves"), emanating from the margins of etch pits, would act to lower the height of the entire surface, thus linking the periodic generation of steps and the overall dissolution rate, and obviating characterization of terraces as "non-reactive". Second, it incorporated the $\Delta G_{\text {crit }}$ parameter to yield a distinct mechanistic divide between rates near $\left(\Delta G_{\text {crit }}<\Delta G<0\right)$ and far from equilibrium $\left(\Delta G<\Delta G_{\text {crit }}\right)$. In the former regime, hollow cores have stabilized, the energy to surmount the barrier to new stepwave formation is not available, and rates are dependent on reactive sites on extant steps. In the latter, sufficient energy is available to activate the entire surface. This mechanistic distinction, discussed more completely by Lüttge [17], also yields the possibility that the observed, instantaneous rate of reaction does not necessarily reflect the coexisting free energy regime, but instead ties the observed rate with the reactive history of the surface. Because many mineral systems of geochemical interest react 
from an initial position far from equilibrium, this underscores a central difficulty in rate measurement and the attendant assumption of steady state [18].

\subsection{Experimental and analytical advances, and the stochastic nature of mineral dissolution kinetics}

The progress in theoretical understanding, particularly the relatively recent insights afforded by the stepwave model, occurred hand in hand with direct measurements of mineral dissolution and growth reactions, using atomic force microscopy (AFM) and vertical scanning interferometry (VSI). Specific application of AFM and VSI to surface reactions of geochemically important minerals (e.g. silicates, carbonates) has been detailed extensively $[19,20]$, and is discussed later in this paper; here we shall primarily enunciate the overall importance of these approaches to the study of mineral dissolution kinetics in general. Both AFM and VSI are now part of a standard approach in geochemistry to mineral reaction rates, and afford direct measurements of the mineral surface. They are often used in complementary fashion. AFM can be used under in situ conditions with a fluid cell, and is capable of essentially atomic or near-atomic resolution under ideal conditions. It is particularly valuable in monitoring the movements of atomic steps, particularly relevant to dissolution at screw dislocations: for example, AFM has been critical in elucidating details of site-specific interaction of dissolved components with mineral surfaces [e.g.,21]. Next generation, ultra-high-resolution frequency modulation AFM (FM-AFM) allows analyzing details of mineral surfaces in breathtaking detail, including 3D mapping of the solid-liquid interface structure itself $[22,23]$. However, AFM is best suited to near-atomically flat surfaces and its practical field of view is limited to a few tens of microns depending on the roughness of the surface. In contrast, VSI offers very high vertical resolution and large fields of view. VSI datasets can be digitally joined to create surface maps covering $\mathrm{mm} 2$ of the mineral surface, but lateral resolution is limited by the objective's numerical aperture [24]. More importantly, VSI offers an unequivocal and unambiguous means of resolving dissolution rate: because dissolution involves overall loss in surface height with progressive material removal over time, those measurements allow a powerful means of resolving rates (change in mass per unit area per unit time). VSI may be used either in situ with fluid [25], (or near in situ [26]), or more typically, ex-situ (true in situ VSI operation requires adequate optical compensation).

Prior to VSI's application, dissolution rates were typically measured by changes in solution composition over time, where the solid and solution were either confined in a closed (batch) system, or the solid was confined but the fluid was allowed to interact by controlled flow or agitation and subsequent removal in reactors described as plug flow, fluidized bed, constantly stirred tank, or variants thereof. The transformation of rate data from concentration changes in the reacted solution (or in some cases, changes in the mass of the solid), involved a normalization to a measure of mineral surface area. The problem with this normalization, now well-recognized, is the complex and heterogeneous distribution (over time and length scales) of reaction rates over the mineral surface. The concept of "reactive surface area", previously mentioned and still popular in the reactive transport model literature, does not represent a viable means of treating this problem, as it entails a fundamental uncertainty (distribution of surface reactivity), and an assumption that is indefensible in defective crystals (constant reactivity). Simply put, "reactive surface area" is a distinction without a difference, and lacks a rigorous definition as well.

However, it is also important to point out that the reproducibility within a powder dissolution dataset may be quite good: e.g., in a compilation of calcite dissolution rate data [27], the variation within a given laboratory's dataset for the alkaline region (where rates are independent of $\mathrm{pH}$ ) is far lower than differences between different datasets measured for a given solution condition, which may reach orders of magnitude. In summary, the calculation of dissolution rates of mineral powders via surface area normalization is thus increasingly recognized as problematic, with as yet no clear path forward.

In addition, as mentioned above in the discussion of free energy dependence, there is recognition that the variation of rates observed during mineral dissolution may also reflect the direction in which equilibrium is approached. If, as is typical, the mineral surface has approached equilibrium from a far-from-equilibrium position, it may still possess reactive topography (excess kinks, reactive step edges) that continue to drive dissolution, although it now resides within the region of hollow core stability. The rate observed under these conditions will thus differ from that predicted from the functional rate equation. This potential for non-steady-state rates is thus a potent source of rate variation [18].

\subsection{New approaches in geochemistry}

The problems described above are of a fundamental nature, and derive less from a lack of experimental data, and more from the inherent difficulty in understanding the role of kinetic mechanism in surface-controlled dissolution reactions for minerals of geochemical interest. In particular, there is a need to understand how these interactions scale: how systems change depending on such parameters as particle size and reaction time. The recognition of intrinsic variation in rate is a key recent finding, and explicit strategies for treatment are still in their infancy $[28,29]$. Yet, ultra-fast kinetics, heterogeneity of intrinsic rates, distribution of crystallite sizes and defect density, and coupling with other mechanisms such as nucleation and growth constitute the characteristic domain of dissolution reactions observed for cement. 


\section{Concepts of mineral dissolution applied to} cementitious systems

\subsection{Experimental Framework}

Portland cement is a synthetic assemblage of several intermixed minerals which all include $\mathrm{CaO}$ as a common base. Among those, alite is the most abundant and therefore often used as a simple model cement. Despite the above described state of the art in geochemistry, the dissolution of $\mathrm{C}_{3} \mathrm{~S}$ was only recently replaced in the general framework of crystal dissolution [2]: like natural minerals, $\mathrm{C}_{3} \mathrm{~S}$ exhibits a high reactivity at far from equilibrium conditions, while its dissolution rate decreases drastically in near-equilibrium conditions. The dependency of reactivity on the saturation state of the surrounding solution has been documented [30], indicating for several $\mathrm{C}_{3} \mathrm{~S}$ polymorphs a trend similar to most natural minerals. This finding has been replicated [31] and corroborated by topological analyses [32].

However, as described above, the solution approach $[30,31,33]$, i.e. continuous monitoring of the evolution of the released ions by inductively coupled plasma atomic emission spectroscopy (ICP-AES) of $\mathrm{C}_{3} \mathrm{~S}$ powders stirred in an 'infinite' volume of solution to avoid any hydrate precipitation, is unspecific (i.e. exposed crystal faces are undifferentiated) and has limitations that preclude the measurement of the specific surface evolution of the dissolving interface, so that only instantaneous dissolution rates can be determined shortly after initial wetting of the sample, when its surface area can be fairly approximated to that of the pristine powder. On the other hand, the topological method [32] using vertical scanning interferometry (VSI) avoids any surface area measurement making the comparison between rates of dissolution more accurate and allows accessing localized morphological information. Nevertheless, due to some lateral resolution limitations of hundreds of nanometers (objective dependent), VSI averages measurements over a 'coarse' unit area (with respect to the atomic resolution of the involved processes).

\subsection{Thermodynamic framework of dissolution}

The dissolution rate of a crystalline material is influenced by its interfacial properties and the surrounding solution through the chemical potential difference as the driving force of dissolution. Interfacial properties reflect the chemical composition, type of bond, crystallographic orientation of the exposed surfaces, impurities incorporated in the crystal, and lattice defects, while solution properties involve the nature of the solvent and its composition, ionic force, temperature, hydrodynamic conditions, and other parameters. The characterization of solution properties is therefore of prime importance for the calculation of saturation indices and consequently under-saturation, reflecting the deviation from equilibrium, expressed as:

$$
\sigma=\frac{\Delta \mu}{k T}=\frac{\Delta G^{*}}{R T}=\ln \left(\frac{\Pi}{K_{s}}\right)
$$

with $\mathrm{k}$ the Boltzmann's constant, $\mathrm{T}$ the absolute temperature, $\Delta \mu$ the chemical potential difference, $R$ the gas constant, $\Delta G^{*}$ the free energy difference between the under-saturated solution and the solution in equilibrium with the solid, that is between $\Pi$, the ion activity product and $\mathrm{K}_{\mathrm{S}}$, the thermodynamic solubility product.

$\mathrm{C}_{3} \mathrm{~S}$ dissolves according to:

$$
\mathrm{C}_{3} \mathrm{~S}+6 \mathrm{H}_{2} \mathrm{O} \rightarrow 3 \mathrm{Ca}^{2+}+\mathrm{H}_{4} \mathrm{SiO}_{4}+6 \mathrm{OH}^{-}
$$

So that:

$$
\Pi=\left\{\mathrm{Ca}^{2+}\right\}^{3} \cdot\left\{\mathrm{H}_{4} \mathrm{SiO}_{4}\right\} \cdot\left\{\mathrm{OH}^{-}\right\}^{6}
$$

This relationship indicates that high concentrations of calcium and hydroxide ions, i.e. conditions usually met within seconds upon cement hydration, lead to high $\Pi$ values, that is, low under-saturation or conditions close to equilibrium. As consequence, the dissolution rate decreases. The determination of $\Pi$ is crucial since the presence of foreign species (ions or organic molecules) can influence the solution chemistry through the formation of complexes which will affect the under-saturation and by extension the rate of dissolution. Determination of $\Pi$ therefore relies on accurate and complete thermodynamic description of the solution to calculate activities through speciation modelling tools such as PHREEQC [34] or GEMS [35].

$\mathrm{C}_{3} \mathrm{~S}$ solubility $\left(\mathrm{K}_{\mathrm{s}}\right)$ could theoretically be determined through its free enthalpy of formation, provided that its surface state is known. Indeed, surface complexes, charging, water, and ionic adsorption can stabilize or destabilize the surface. In their original determination, Stein and Stevels [36] neglected these surface reactions. This was a key source of error in many subsequent works, which invoked the putative formation of a metastable hydrate membrane [36,37,38], instead of the partial hydroxylation of the $\mathrm{C}_{3} \mathrm{~S}$ surface [34], to justify the discrepancy between the calculated solubility and the low hydration rates observed. Alternatively, Nicoleau et al. [30] tried determining an experimental solubility product using a kinetic approach, showing by the way that the surface stability was $\mathrm{pH}$ dependent. This highlighted the importance of surface hydroxylation, recently supported by NMR [35]. Nevertheless, this value depends on interfacial properties which so far remain experimentally inaccessible. The use of $\ln \Pi$ instead of $\ln \left(\Pi / K_{s}\right)$ is therefore preferred because of this inherent uncertainty regarding the true solubility.

\subsection{Mechanism and topology of $\mathrm{C}_{3} \mathrm{~S}$ dissolution}

Figure 1 shows that $\mathrm{C}_{3} \mathrm{~S}$ dissolution patterns are similar to most natural mineral, with a more or less marked signature for all polymorphs and faces: the dissolution rate generally significantly decreases with under-saturation. As mentioned earlier this decrease was understood through the stepwave model, which supposes two distinct mechanisms at high and low under-saturation. The theory of crystal dissolution foresees that it should initiate at areas of excess surface energy usually provided by lattice deformations [39]. In the case of alite, the transition is more visible when measured on flat polished surfaces, whereas measurements of discrete 
powders integrate a distribution of rates that likely obscure this distinct transition, and provide an apparent linearity. For these reasons as well as those described above, this transition most probably does not corresponds to a sudden change of exclusive mechanism.

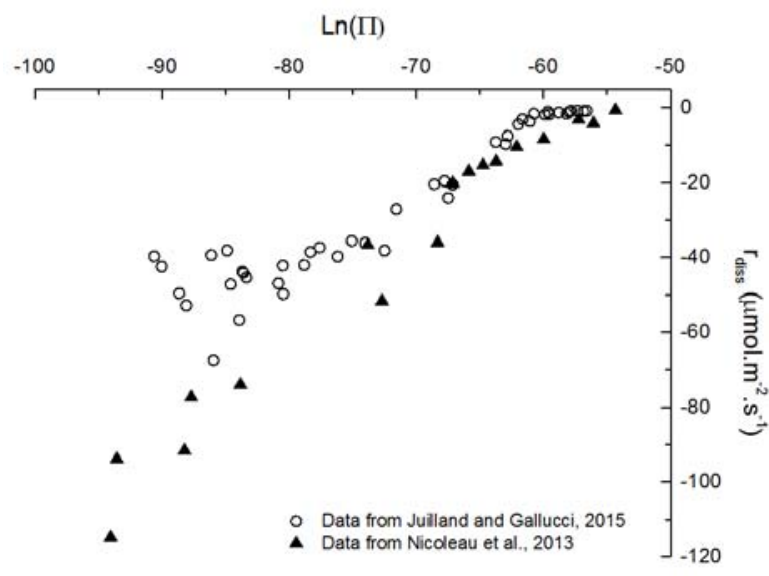

Figure 1. Comparison of dissolution rates from Nicoleau et al. [30] obtained from bulk powder dissolution in stirred batch reactors and Juilland and Gallucci [32] obtained by VSI measurements (values obtained at room temperature and with at flow rate close to 36 $\mathrm{mL} / \mathrm{min} / \mathrm{mm}^{2}$ ). All $\operatorname{Ln}(\Pi)$ values were calculated according to the Phreeqc database taking into account the calcium silicate complexes recently determined [33] leading to values slightly different from the original published data.
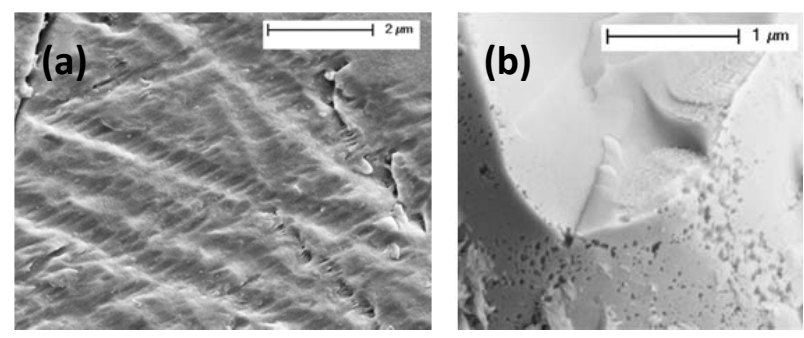

Figure 2.(a) Dissolution front of polished section of alite exposed $1 \mathrm{~h}$ to a solution at $18 \mathrm{mM} \mathrm{Ca} 2+$ and $\mathrm{pH}=12.6$. Shallow etch pits can be observed across the all surface and follows lines of defects induced by sample polishing. (b) Alite surface of a hydrated grain during the acceleration period. The surface presents large pitting areas. In both cases, the undersaturation conditions with respect to alite are in a regime of slow rate of dissolution when etch pits should not be active.

Processes involved in cement hydration are extremely fast, and the nearly instantaneous build-up of ions in the solution upon dissolution of alite makes it difficult to discriminate the various regimes. In particular, the system proceeds from far from- to near-equilibrium conditions within seconds, so that $\Delta G_{\text {crit }}<\Delta G$ at nearly all reaction times, and dissolution is expected to proceed through step retreat only. Indeed, etch pits are still observed under near-equilibrium conditions at early stages [32] (Figure 2a), as well after hours of hydration [30] (Figure 2b). This observation indicates evidences that, in the case of alite, etch pits continuously open up and grow in all ranges of under-saturation, provided that the deviation from equilibrium is locally high-enough. Growth rate of etch pits is may also be driven by strong, localized undersaturation, possibly controlled by the distribution of precipitating hydrates. The precipitation of portlandite can favor such high deviations from equilibrium, acting as a local sink for calcium and hydroxide ions, and generating significant decrease in concentration [40].

\subsection{Towards dissolution in cementitious systems: Intrinsic and extrinsic factors}

The several steps involved in the production process of cement impact its intrinsic reactivity: foreign atoms present in the raw mix extracted from quarries or chemicals brought in the kiln through the use of alternative fuels strongly impact its polymorphic assemblage, as well as the type and density of crystalline defects [41]. Quenching from $1500^{\circ} \mathrm{C}$ to prevent monoclinic alite ripening into more stable phases also yields stresses in the crystal structure, relaxed through the appearance of dislocations; crystals are further subjected to plastic deformation during milling. Although controlled in any other industrial context, the in-depth analysis of the defect-reactivity relation has not been performed for alite. However, the impact of thermal treatments or annealing, supposedly reducing defect density, has clearly evidenced an effect on dissolution and hydration [3, 42]. In particular, thanks to the enhanced diffusion of atoms in the crystal structure, annealing lowers reactivity through annihilating a fraction of the defects and reducing the energy of others (Figure 3a): pairing of vacancies and interstitial ions, clustering of foreign atoms, annihilation or stacking of dislocations [43].

Surface properties may also be strongly impaired by the adsorption of species coming from the solution. The scarce data available from the literature does not allow a classification per se but gives trends on the type and strength of interfacial interactions and the associated decrease of dissolution rates. Weak interactions (e.g. Hbonds) show little or no impact on the dissolution rate, as observed with polyacrylamide surface-functionalized lattices [5]. Monovalent ions hardly interact with $\mathrm{C}_{3} \mathrm{~S}$ and barely show any effect on dissolution. Species with higher valences such as sulfates [33], phosphates [44] or comb-copolymers [42], which interact electrostatically with the charged $\mathrm{C}_{3} \mathrm{~S}$ surface, are able to slow down the dissolution rate more or less effectively depending on their charge density. Covalent interactions are expected to impair dissolution more strongly: for instance, aluminum chloride significantly reduces $\mathrm{C}_{3} \mathrm{~S}$ dissolution rate in alkaline conditions $(\mathrm{pH} 12)$ [33], which can be attributed to the formation of covalent SiO-Al bonds $[45,46]$. Such chemical or physical interactions would particularly stabilize kink [39] atoms which are key in dissolution kinetics [47]. Strong interactions can completely suppress activity at dissolution etch-pits, while weaker interactions can at least induce a change in the pit shape (reflecting a change in relative step velocities), resulting in diminished taper (Figure 3b) [48]. 


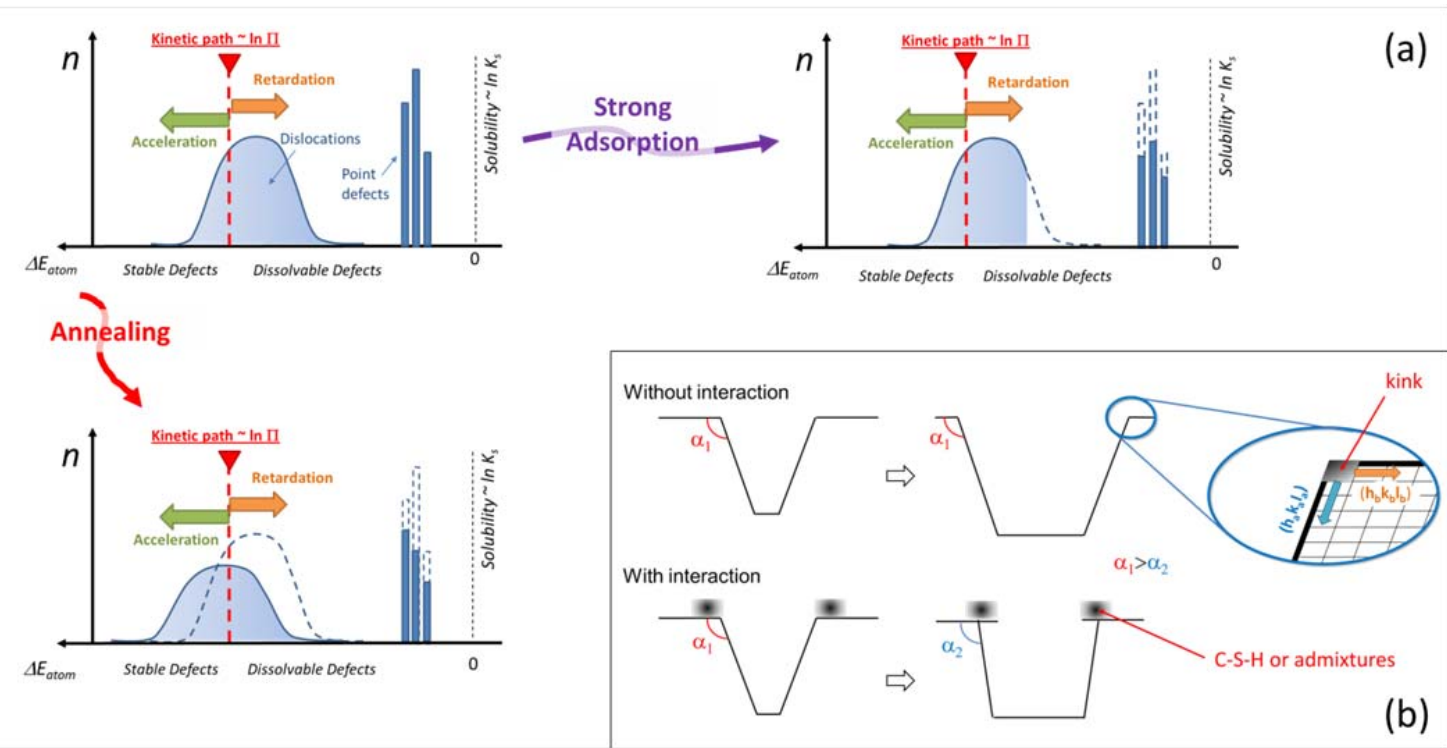

Figure 3. (a) Theoretical energy distribution of defective surface atoms and its evolutions after either being partly annealed or in case of strong adsorption of admixture on high-energy defects. The energy excess of each atom is given by the difference between the atom energy in equilibrium with the solution and the current atom energy: $\Delta E_{\text {atom }}=E_{\text {eq }}-E_{\text {atom. }}$. The capacity of being dissolved or not for an atom is given by the position of the kinetic path. If the kinetic path (given by the $\operatorname{Ln}(\Pi)$ value) is close to the solubility (given by the $\ln K_{s}$ value), only few atoms are enough out-of-equilibrium to be dissolved. Any means accelerating the C-S-H precipitation will shift the position of the kinetic path even farther from the $\mathrm{C}_{3} \mathrm{~S}$ solubility equilibrium and more defects are likely to dissolve, and vice versa. (b) Sketch capturing the growth of a pit in the absence and in the presence of a strong interaction at the interface close to the etch-pit, coming for instance either from the adsorption of an organic admixture or due to the precipitation of C-S-H. The interaction stabilizes the surface and will reduce the dissolution in the $\left(\mathrm{h}_{b} \mathrm{k}_{b} \mathrm{l}_{b}\right)$ direction. It will result in a change of the pit shape when the pit expansion meets the C-S-H crystallite or the admixture.
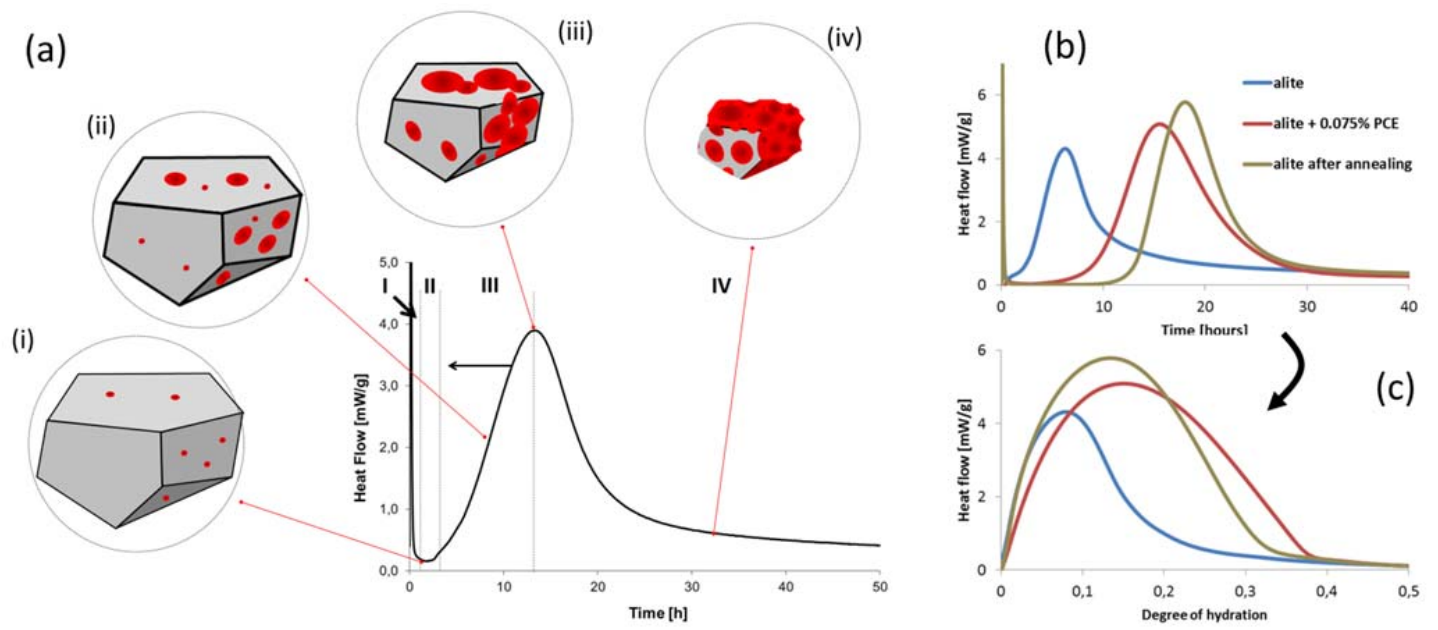

Figure 4. (a) Typical evolution of the heat flow released during the hydration of alite and diced into different steps: I pure dissolution, II the lowactivity period, III the accelerating step and IV the decelerating period. Note that the heat flow is proportional to the hydration rate. The curve is decorated with schematic drawings showing the expected development of an alite grain with respect to the sole dissolution viewpoint: (i) first nucleation of etch-pits, (ii) expansion of etch-pits, (iii) start of the etch-pits coalescence and (iv) full surface coverage by etch-pits. The grain also shrinks because of the dissolution by step-retreat. We intentionally show different reactivity for the different faces since different crystals have to be exposed to the solution and reactivity deviations have been highlighted for those latter [56]. (b) Heat flow released during the hydration of $\mathrm{C}_{3} \mathrm{~S}$ in the presence of a typical superplasticizer and after annealing $\left(6 \mathrm{hrs} @ 650^{\circ} \mathrm{C}\right)$. (c) Same experiments as in (b) but heat flows are plotted as a function of the $\mathrm{DoH}\left(\Delta \mathrm{H}_{\text {hyd }}=500 \mathrm{~J} / \mathrm{g}\right)$. This way highlights the evolution of the dissolution rate upon dissolution and above all at which $\mathrm{DoH}$ the maximum rate is reached. 


\section{Implications of dissolution for cement hydration}

\subsection{Hydration kinetics of alite in cements}

The hydration kinetics of cement are generally described as the succession of 4 steps (Figure 4a): (I) the pure dissolution step, (II) the dormant period, (III) the acceleration phase and (IV) the deceleration phase. More than the steps themselves, the transitions between them have always been the source of focused attention and controversy within the scientific community. After decades of fruitless debates, two major questions remain unanswered: 1) which processes govern the dormant period, and 2) what is the origin of deceleration? Beyond a scientific dimension, the answers to those questions are of industrial importance as the key lock to the rational tailoring of early hydration and its associated development of mechanical properties.

The geochemical concepts, introduced only recently to the cement community, challenge the old vision that C-S-H nucleation, growth and impingement of crystallites, completely control hydration kinetics. This model lacks the support of any single unambiguous observation, and furthermore neglects the central mass balance constraint that links $\mathrm{C}-\mathrm{S}-\mathrm{H}$ precipitation and $\mathrm{C}_{3} \mathrm{~S}$ dissolution reactions, as strictly coupled rates must be equivalent. Therefore, any mechanism, process or kinetic event ascribable to C-S-H precipitation has a counterpart in $\mathrm{C}_{3} \mathrm{~S}$ dissolution. Indeed, some have been identified.

\subsection{Dormant period}

Prior to thorough study [30], alite solubility was considered to be very high, whereas the discrepancy between putative solubility and measured ionic concentrations in the pore solution pointed to a central inconsistency. This observation supported the hypothesis of the formation of a protective hydrated layer, whose role was to retard the rate of alite dissolution [49]. Advances in geochemistry helped support the notion that typical pore solutions are indeed close to the apparent experimental solubility of alite, explaining why its dissolution rate drastically decreases few seconds/minutes after the first contact with water. In parallel, it was observed that protonation of silicate monomers and free oxygen atoms strongly stabilize alite surface and reduce its solubility $[30,50]$. As mentioned earlier, the surface bonding of ions $[33,51]$ or the adsorption of polymers [42] also stabilize the alite surface and in turn extend the dormant period. Although mechanisms have not been resolved, the analogy with the hydration of annealed alite suggests a site specific interaction [42], without any impact on the subsequent acceleration rate (Figure $4 \mathrm{~b}$ ): adsorbed ions and molecules passivate the surface and block the dissolution of line defects, like dislocations outcropping at the surface, or point defects.

\subsection{Acceleration regime}

This step shows an accelerating reaction rate, i.e. both $\mathrm{C}-\mathrm{S}-\mathrm{H}$ precipitation and $\mathrm{C}_{3} \mathrm{~S}$ dissolution rates have to increase. This is trivially accepted for nucleation and free growth using exponential laws [52] with the success of the Johnson-MehlAvrami-Kolmogorov model and its derivatives (see [53]) which imply an accelerative rate. This is far less obvious for dissolution since the dissolving surface area $S_{d}$ is expected to decrease with the shrinking of the dissolving grains, implying that sufficient increase in under-saturation should produce equivalent increase in $S_{d} \cdot R_{\text {int }}\left(R_{\text {int }}\right.$ is the interfacial dissolution rate). However, this is not observed, as under-saturation hardly changes [54] over this interval, which implies an increase in either the number of dissolution sites or their surface area. The dissolution theory as introduced above offers ways to envisage the increase of dissolving surface, even at low under-saturation; for example, via the activation of kinks in the vicinity of screw-dislocations: their dissolution and expansion in the capillaries of hollow cores (Figure 4a.ii) would increase the dissolving surface and the overall dissolution rate as a consequence.

\subsection{Deceleration regime}

Above 10 to $20 \% \mathrm{DoH}$, the hydration rate slows down nearly as rapidly as it had accelerated. This decline was long ascribed to the impingement of C-S-H crystallites growing at the surface of alite grains or outwards in the solution. However this idea can be challenged by hydration in highly diluted suspensions, in which the inter-grain space is large enough to avoid impingement, and in which C-S-H precipitation is shifted away from the alite surface into the solution (discussion in [55]). Furthermore, annealing or the use of plasticizers similarly increase the DoH at maximum rate (Figure $4 b, c$ ), an observation that is hardly reconcilable with pure nucleation and growth kinetics. Here again the dissolution theory brings an alternative view through a decreased dissolution rate, due either to the overall shrinkage of grains (reduced $R_{\text {int }}$ ), or because etch pits have grown to the extent where they coalesce, resulting in lower overall surface (reduced $S_{d}$ ). In both instances $S_{d} \cdot R_{\text {int }}$ decreases (Figure 4a.iii) below a critical value, driving deceleration. A lack of proper data prevents discriminating those two hypotheses. However, an isotropic shrinkage model cannot alone describe the experimental dissolution curve [56], and would imply a constant DoH at maximum rate, which is not observed, while the coalescence of etch pits is already microstructurally evidenced e.g. [57]. At later reaction times, upon completion of pit coalescence, the roughness of alite surface is stable and $S_{d} \cdot R_{\text {int }}$ decreases at a steady state mainly due to the grains shrinkage (Figure $4 a . i v)$ [58].

\section{Oversaturation of etch pit-falls. A perspective}

Over the last decade, new dissolution-based concepts have allowed a fundamental reconsideration that casts alite 
dissolution as the driving force of cement hydration. Indeed, all approaches to explain hydration so far can be revisited and soundly supported from a dissolution perspective. Nevertheless, describing alite dissolution in real cement systems must reconcile the coupled interaction of two processes: the composition of the pore solution which controls the interfacial dissolution rate through the deviation from equilibrium, and the surface energy at the crystal level which governs the evolution of surface topography. The general experimental difficulty in decoupling surface topography and energetics is exacerbated for alite because of the limited size of crystallites and etch-pits, its high dissolution rate compared to other minerals, and the parallel precipitation of hydrates of the same chemical composition in the vicinity of the dissolving areas. Furthermore, the lack of proper characterization of the nature and density of defects in alite polymorphs and the missing information regarding the energetics of crystal faces exposed to the solution remains experimentally and scientifically challenging. Increasingly, the approach taken in understanding problems of crystal dissolution involves a recursive, reciprocal process of measurement and analysis of rates coupled with computer simulation of virtual crystals (e.g., via kinetic Monte Carlo techniques [59]). Dissolution rates and their surface distribution measured in the laboratory have to be compared with the results of simulations parameterized by e.g., DFT-acquired bond hydrolysis activation energies. This comparison affords a check on the distribution of reaction mechanism, as reaction paths that are inadequately or inaccurately described in the simulation will produce poor agreement with measured results. The comparison of parameterized model simulation and measured experimental results also yields key information as to the variation of rates over the surface and over time. This implies the development of adequate numerical tools which, although initiated for cement [60], will most probably require more substantial time and effort.

\section{References}

[1] M. Costoya, Effect of particle size on the hydration kinetics and microstructural development of tricalcium silicate EPFL Thesis 4102 Lausanne, (2008)

[2] P. Juilland et al. Dissolution theory applied to the induction period in alite hydration Cement and Concrete Research (2010) 40(6) 831-844.

https://doi.org/10.1016/j.cemconres.2010.01.012

[3] P. Fierens, Y. Kabuema, J. Tirlocq, Influence de la température de recuit sur la cinétique d'hydratation du silicate tricalcique Cement and Concrete Research (1982) 12(4) 455-462 https://doi.org/10.1016/0008-8846(82)90060-6

[4] P. Fierens, Y. Kabuema, J. Tirlocq, Influence du milieu de trempe sur la cinétique d'hydratation du silicate tricalcique, Cement and Concrete Research (1982) 12(2) 191-198 https://doi.org/10.1016/0008-8846(82)90006-0

[5] L. Nicoleau, Interactions physico-chimiques entre des latex et les phases principales du ciment, Uni. Burgundy Thesis, Dijon, (2004)

[6] J.W. Bullard et al., A critical comparison of 3D experiments and simulations of tricalcium silicate, Journal of the American Ceramic Society, (2010) v.100. https://doi.org/10.1111/jace.15323
[7] D. J. Barber et al. Dislocations in minerals. In: J. P.HIRTH, L. K. Dislocations in Solids. Vol. 16. [S.I.]: Elsevier, (2010) 171-232.

[8] F.C. Frank, Capillary equilibria of dislocated crystals. Acta Crystallographica (1951) 4, (6) 497-501. https://doi.org/10.1107/S0365110X51001690

[9] A. J. Gratz, P. E. Hillner, P. K. Hansma, Step dynamics and spiral growth on calcite. Geochimica et Cosmochimica Acta (1993) 57 491-495, 1993.

[10] C. Klemenz, Hollow cores and step-bunching effects on (001)YBCO surfaces grown by liquid-phase epitaxy. Journal of Crystal Growth (1998) 187221 - 227.

https://doi.org/10.1016/S0022-0248(97)00866-X

[11] M. Plomp, K. Maiwa, W. J. P. V. Enckevort, Atomic force microscopy observations of hollow cores on the 111 and 100 faces of barium nitrate. Journal of Crystal Growth (1999) 198199 246-252. https://doi.org/10.1016/S0022-0248(98)01103-8

[12] W. K. Burton, N. Cabrera, F. C. Frank, The growth of crystals and the equilibrium structure of their surfaces. Philosophical Transactions of the Royal Society of London. Series A, Mathematical and Physical Sciences, (1951) 243 (866) 299-358. https://doi.org/10.1098/rsta.1951.0006

[13] N. Cabrera, M. M. Levine, XLV. On the dislocation theory of evaporation of crystals. Philosophical Magazine (1956) 1 (5) 450458. https://doi.org/10.1080/14786435608238124

[14] B. Van der Hoek, J. P.Van der Eerden, P.Bennema, Thermodynamical stability conditions for the occurrence of hollow cores caused by stress of line and planar defects. Journal of Crystal Growth, (1982) 56 621-632. https://doi.org/10.1016/0022-0248(82)90045-8

[15] A. C. Lasaga, A. E. Blum, Surface chemistry, etch pits and mineralwater reactions. Geochimica et Cosmochimica Acta (1986) 50 (10) $2363-2379$.

https://doi.org/10.1016/0016-7037(86)90088-8

[16] A. C. Lasaga, A. Luttge, Variation of crystal dissolution rate based on a dissolution stepwave model. Science (2001)291 (5512) 2400-2404. https://doi.org/10.1126/science.1058173

[17] A. Lüttge, Crystal dissolution kinetics and Gibbs free energy. Journal of Electron Spectroscopy and Related Phenomena (2006) 150 248-259. https://doi.org/10.1016/j.elspec.2005.06.007

[18] R. S. Arvidson, A. Luttge, Mineral dissolution kinetics as a function of distance from equilibrium - New experimental results. Chemical Geology (2010) 269 79-88. https://doi.org/10.1016/j.chemgeo.2009.06.009

[19] I. Kurganskaya et al., Does the stepwave model predict mica dissolution kinetics? Geochimica et Cosmochimica Acta (2012) 97 120-130. https://doi.org/10.1016/i.gca.2012.08.033

[20] R. S. Arvidson, J. W. Morse, Formation and diagenesis of carbonate sediments. In: HOLLAND, H. D.; TUREKIAN, K. K. Treatise on Geochemistry. [S.I.]: Elsevier (2014) 9.3. Cap. 7 61101. https://doi.org/10.1016/B978-0-08-095975-7.00703-8

[21] E. Ruiz-Agudo,; C. V. Putnis, Direct observations of mineral-fluid reactions using atomic force microscopy: the specific example of calcite. Mineralogical Magazine (2012) 76 (1) 227-253. https://doi.org/10.1180/minmag.2012.076.1.227

[22] H. Söngen et al., Three-dimensional atomic force microscopy mapping at the solid-liquid interface with fast and flexible data acquisition. Review of Scientific Instruments (2016) 87, 0637041-063704-4. https://doi.org/10.1063/1.4952954

[23] M. Nalbach et al. Molecular self-assembly versus surface restructuring during calcite. Langmuir (2016) 32 9975-9981. https://doi.org/10.1021/acs.langmuir.6b02685

[24] R. S. Arvidson, et al. Lateral resolution enhancement of vertical scanning interferometry by sub-pixel sampling. Microscopy and Microanalysis (2014) 20 (1) 90-98. https://doi.org/10.1017/S1431927613013822

[25] S. Ueta et al., Dynamic and topographic observation of calcite dissolution using enhanced in-situ phase-shift interferometry. Journal of Crystal Growth (2013) 363 294-299. https://doi.org/10.1016/i.jcrysgro.2012.11.021

[26] I. Bibi et al., Non steady-state calcite dissolution controlled by complex surface geometries. Minerals, (2018) in review.

[27] R. S. Arvidson et al., Variation in calcite dissolution rates: A fundamental problem? Geochimica et Cosmochimica Acta (2003) 67 1623-1634 2003.

[28] C. Fischer, R.S. Arvidson,A. Luttge, How predictable are dissolution rates of crystalline material? Geochimica et Cosmochimica Acta (2012) 98 177-185 https://doi.org/10.1016/j.gca.2012.09.011 
[29] A. Lüttge, R.S. Arvidson, C. Fischer, A stochastic treatment of crystal dissolution kinetics. Elements (2013) 9 (8) 183-188. https://doi.org/10.2113/gselements.9.3.183

[30] L. Nicoleau, A. Nonat, D. Perrey, The di- and tricalcium silicate dissolutions. Cement and Concrete Research (2013) 47 14-30. https://doi.org/10.1016/j.cemconres.2013.01.017

[31] F. Bellmann et al., Dissolution rates during the early hydration of tricalcium silicate. Cement and Concrete Research (2015) 72(Supplement C) 108-116.

https://doi.org/10.1016/j.cemconres.2015.02.002

[32] P. Juilland, E. Gallucci, Morpho-topological investigation of the mechanisms and kinetic regimes of alite dissolution. Cement and Concrete Research (2015). 76 (Supplement C) 180-191. https://doi.org/10.1016/j.cemconres.2015.06.001

[33] L. Nicoleau, E. Schreiner, A. Nonat, Ion-specific effects influencing the dissolution of tricalcium silicate. Cement and Concrete Research (2014) 59(Supplement C) 118-138 2014.

[34] D.L. Parkhurst, C.A.J. Appelo, User's guide to PHREEQC - a computer program for speciation, reaction-path, 1D-transport, and inverse geochemical calculations, U.S. Geol. Survey WaterResour. Invest. Rep (1999) 99-4259

[35] D. Kulik, U. Berner, E. Curti, Modelling chemical equilibrium partitioning with the GEMS-PSI code, PSI Scientific Report IV (2003) 109-122 (http://les.web.psi.ch/Software/GEMS-PSI/).

[36] H.N. Stein, J.M. Stevels, Influence of silica on hydration of 3CAO,SiO2. Journal of Applied Chemistry (1964)14 (8) 338. https://doi.org/10.1002/jctb.5010140805

[37] D.L. Kantro, S. Brunauer, C.H. Weise, Development of surface in hydration of calcium silicates .2. Extension of investigations to earlier and later stages of hydration. Journal of Physical Chemistry (1962) 66 (10) 1804 https://doi.org/10.1021/j100816a007

[38] E.M. Gartner, H.M. Jennings, Thermodynamics of calcium silicate hydrates and their solutions. Journal of the American Ceramic Society (1987) 70 (10) 743-749.

https://doi.org/10.1111/j.1151-2916.1987.tb04874.x

[39] K. Sangwal, Etching of Crystals - Theory, Experiment and Application. Defects in Solid, ed. J.N. S. Amelinckx. NorthHolland (1987) 15

[40] J.W. Bullard, R.J. Flatt, New insights into the effect of calcium hydroxide precipitation on the kinetics of tricalcium silicate hydration, Journal of the American Ceramic Society (2010) 93 (7) 1894-1903. https://doi.org/10.1111/j.1551-2916.2010.03656.x

[41] H.F.W. Taylor, Cement Chemistry 2nd Ed. Thomas Telfort Publishing, London (1997). https://doi.org/10.1680/cc.25929

[42] D. Marchon et al., Molecular and submolecular scale effects of comb-copolymers on tri-calcium silicate reactivity: Toward molecular design. Journal of the American Ceramic Society (2017) 100 (3) 817-841. https://doi.org/10.1111/jace.14695

[43] J.L. Martin, Dislocations et plasticité des cristaux, Presses Polytechniques et Universitaires Romandes (2000) 67-86

[44] P. Benard, Etude de l'action des phosphates presents dans l'eau de gachage sur I'hydratation d'un ciment Portland, Uni. Bourgogne Thesis, Dijon (2005)

[45] T. Chappex, K.L. Scrivener, The Effect of Aluminum in Solution on the Dissolution of Amorphous Silica and its Relation to Cementitious Systems, Journal of the American Ceramic Society (2013) 96 (2) 592-597

[46] R.K. Iler, Effect of adsorbed alumina on the solubility of amorphous silica in water, Journal of Colloid and Interface Science (1973) 43 (2) 399-408. https://doi.org/10.1016/0021-9797(73)90386-X

[47] A.C. Lasaga, Kinetic justification of the solubility product: Application of a general kinetic dissolution model, Journal of Physical Chemistry B (2005) 109 1635-1642. https://doi.org/10.1021/jp047631q

[48] A.J. Nijdam, Anisotropic wet-chemical etching of silicon pits, peaks, principles, pyramids and particles, Thesis University of Twente, Enschede, the Netherlands (2001)

[49] E.M. Gartner, J.M. Gaidis, Hydration Mechanisms I. In: Skalny, J.P., Ed., Materials Science of Concrete, The American Ceramic Society, Westerville, (1989) 95-125

[50] E. Pustovgar et al., Understanding silicate hydration from quantitative analyses of hydrating triclcium silicates, Nature Communications (2016) 710952. https://doi.org/10.1038/ncomms10952
[51] P. Suraneni, R.J. Flatt, Use of micro-reactors to obtain new insights into the factors influencing tricalcium silicate dissolution, Cement and Concrete Research (2015) 78 B 208-215.

[52] M. Fanfoni, M. Tomellini, The Johnson-Mehl- AvramiKohnogorov model: A brief review, II Nuevo Cimento D (1998) 20(7-8) 1171-1182. https://doi.org/10.1007/BF03185527

[53] J.J. Zheng, J. Zhang, G.W. Scherer, Prediction of the degree of hydration at initial setting time of cement paste with particle agglomeration, Cement and Concrete Research (2012) 42 12801285. https://doi.org/10.1016/j.cemconres.2012.05.020

[54] L. Nicoleau, A. Nonat, A new view on the kinetics of tricalcium silicate hydration, Cement and Concrete Research (2016) 86 184. https://doi.org/10.1016/i.cemconres.2016.04.009

[55] L. Nicoleau, The C3S dissolution, a determinig kinetic fcator during hydration, Proceedings of the 14th ICCC, Beijing China (2015).

[56] V. ROBIN et al., An outlook into the dissolution anisotropy of tricalcium silicate (2018) To be submitted.

[57] E. Gallucci et al., Microstructural development of early age hydration shells around cement grains, Cement and concrete Research (2010) 40 (1) 4-13. https://doi.org/10.1016/j.cemconres.2009.09.015

[58] L. Nicoleau, M.A. Bertolim, Analytical model for the alite dissolution topography, Journal of the American Ceramic Society (2016) 99 (3) 773-786. https://doi.org/10.1111/jace.13647

[59] I Kurganskaya, A. Luttge, Kinetic Monte Carlo simulations of silicate dissolution: Model. Journal of Physical Chememistry C (2013) 117 24894-24906. https://doi.org/10.1021/jp408845m

[60] E. Durgun, H. Manzano, R.J.M. Pellenq, J.C. Grossman, Understanding and controlling the reactivity of the calcium silicate phases from first principles, Chemistry of Materials (2012) 24 1262. https://doi.org/10.1021/cm203127m 